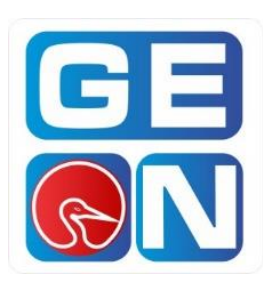

Revista GEON (Gestión, Organizaciones y Negocios.) ISSN: 2346-3910 en línea

revistageon@unillanos.edu.co

Universidad de los Llanos

Colombia

Llenque Tume, Santos Felipe.

Homologación para el fortalecimiento de las micro y pequeñas empresas proveedoras según ISO 9001 de Sider

Perú-Gerdau, Chimbote, Perú

Revista GEON, Vol. 5, No. 2, 2018

Pág. 8-23

Disponible en: $\underline{\text { https://doi.org/10.22579/23463910.77 }}$

Esta publicación se encuentra bajo licencia: Creative Commons

ReconocimientoNoComercial-

SinObraDerivada 4.0 Internacional
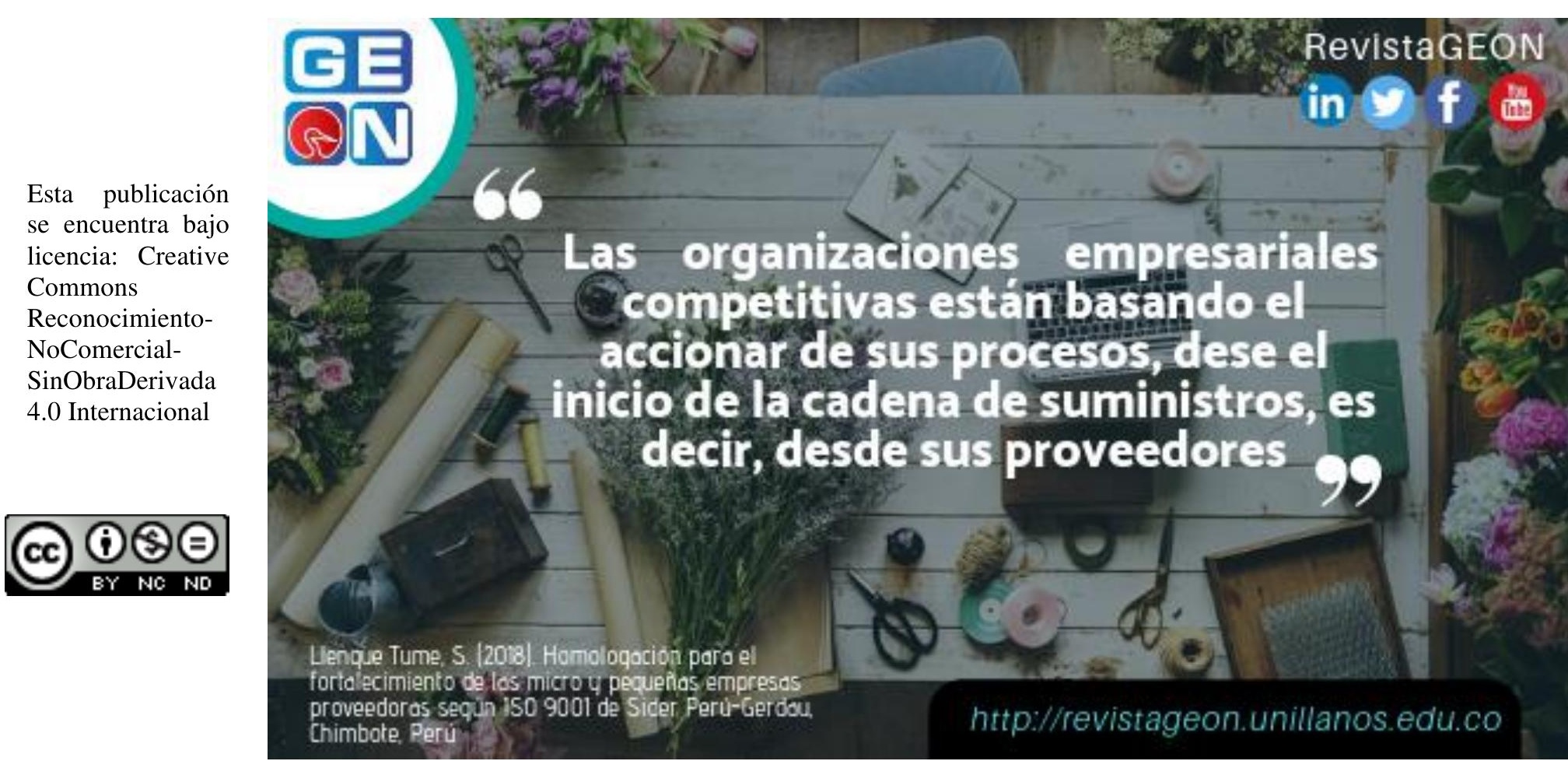


\title{
Homologación para el fortalecimiento de las micro y pequeñas empresas proveedoras según ISO 9001 de Sider Perú-Gerdau, Chimbote, Perú
}

\section{Homologation for the strengthening of micro and small enterprises supplying according to ISO 9001 Sider Peru-Gerdau, Chimbote, Peru}

\author{
Santos Felipe Llenque Tume \\ felipellen2002@yahoo.com
}

Doctor en Administración, Universidad Católica los Ángeles de Chimbote, Perú

Revista GEON Vol. 5 No 2 julio - diciembre 2018

ISSN 2346-3910 en línea http://revistageon.unillanos.edu.co/index.php/geon/article/view/77

Artículo recibido 2018/05/13 aceptado 2018/06/12 


\title{
Resumen
}

El presente trabajo de investigación tuvo como objetivo general, determinar el cumplimiento de los criterios de calificación de los Niveles de Homologación que deben tener las pymes proveedoras de Sider PerúGerdau, Chimbote, 2016. El diseño del estudio fue no experimentaltransversal. Se utilizó una población muestral de 45 empresas que se les aplicó un cuestionario, obteniendo los siguientes resultados: de las 45 empresas que ingresaron al primer Nivel el $100 \%$ cumplieron con todos los criterios de calificación. De las 45 empresas que ingresaron al Segundo Nivel, el 58\% cumplieron con los criterios de calificación. Del total de empresas que ingresaron al Tercer Nivel, el 92\% cumplieron con los criterios de calificación. Concluyendo que, si se cumplen con todos los criterios de calificación de homologación, las empresas quedan expeditas para ser consideradas proveedoras de Sider Perú.

Palabras Clave: Micro y pequeñas Empresas, Homologación, Niveles de homologación, Perú, ISO 9001

\begin{abstract}
The general objective of this research was to determine the fulfillment of the qualification criteria of the Homologation Levels that the SME providers of Sider Perú-Gerdau, Chimbote, 2016 should have. The design of the study was non-experimental-transversal. A sample population of 45 companies was used and a questionnaire was applied, obtaining the following results: of the 45 companies that entered the first level, $100 \%$ met all the qualification criteria. Of the 45 companies that entered the Second Level, 58\% met the qualification criteria. Of the total number of companies that entered the Third Level, 92\% met the qualification criteria. Concluding that, if all the qualification criteria of homologation are met, the companies are expedited to be considered suppliers of Sider Peru.
\end{abstract}

Palabras Clave: Micro and small business, homologation, levels of homologation, Peru, ISO 9001

\section{Code JEL: M1 Business Administration}

\section{Introducción}

En el presente trabajo se esboza el Programa de Desarrollo de Proveedores llevado a cabo por una de las empresas líderes en la producción de aceros largos en el continente americano y una de las principales proveedoras de aceros largos especiales del mundo, como es Sider PerúGerdau, quien se encuentra ubicada en la ciudad de Chimbote, región Ancash. Es a 
través de esta empresa que en alianza estratégica con la institución Asociación Laboral para el Desarrollo, ADEC-ATC, organización gubernamental sin fines de lucro, encargada de llevar a cabo el diagnóstico, capacitación, consultoría, auditoría y la certificación respaldada por CERPER, a través del programa CERTIPYME, quienes desde el año 2012, vienen colaborando para desarrollar el nivel competitivo de las empresas proveedoras de Sider Perú.

Tradicionalmente, en nuestro país, solo han sido las grandes empresas quienes les han dado importancia el proceso de homologación a sus proveedores. Sin embargo, debido a la alta competitividad que existen en los mercados nacionales e internacionales, Sider Perú-Gerdau, se ve en la imperiosa necesidad de implementar estrategias de mejora continua para desarrollar la oferta de empresas locales, fundamentalmente microempresas, y crear confianza en el nivel de cumplimiento, calidad, cantidad y así aprovechar las oportunidades generadas en el mercado.

Según la Superintendencia Nacional de Administración Tributaria-SUNAT, en la Región Ancash, existen un total de 39,206 microempresas, de las cuales el $58 \%$ se encuentran en la provincia de Santa y de este universo, el $40 \%$ se encuentra en el distrito de Chimbote, un $10 \%$ en el distrito de Nuevo Chimbote y el $8 \%$ en el resto de los distritos de la provincia. Se puede decir que, en el distrito de Chimbote, existen aproximadamente 9,095 microempresas y en Nuevo Chimbote unas 2,750 microempresas. Sin embargo, una de las características de las microempresas de nuestro país y, en especial, de la provincia del Santa, donde se encuentra afincada una de las plantas de acero de Sider PerúGerdau, es la alta tasa de informalidad de las mypes, aproximadamente un $70 \%$ de informalidad. Lo que hace que necesariamente aquellas empresas proveedoras de bienes y servicios a Sider Perú- Gerdau, tengan que ser homologadas y certificadas de acuerdo con las normas del programa CERTIPYME.

La empresa Sider Perú-Gerdau considera que es necesario entender tan bien los negocios de sus clientes como los de sus propias empresas que se encuentran distribuidas en 14 países. Además, entiende que sus proveedores son fundamentales para que ella logre alcanzar metas de calidad, seguridad y excelencia. Por eso, selecciona y desarrolla cuidadosamente sus proveedores - y exige que ellos sigan prácticas responsables respecto a las personas y al medio ambiente. Por ese motivo, se está implementando una solución de gestión global del proceso de compras y de la relación con los proveedores lo que le traerá, entre otros beneficios, un cambio en el flujo de informaciones entre Gerdau y sus proveedores en todo el mundo. Considerando que en nuestro país el universo de las empresas formales está conformado en un $98.5 \%$ por micro, pequeñas y medianas empresa (Mipyme) lo que hace que se considere un segmento bastante dinámico en cuanto a sus tasas de creación y cierre, sin embargo, es muy heterogéneo en cuanto a sus características y desempeño. 
Esto hace que no sea adecuado pensar en las Mipyme como un conjunto uniforme de unidades productivas y menos pretender que las estrategias para incentivar su crecimiento sean igualmente efectivas cuando se implementan sin tener en cuenta sus particularidades. Entonces para Sider Perú-Gerdau, existe la necesidad capacitar y certificar a las pequeñas empresas de Chimbote para ingresar en la cadena de suministros de la empresa. En tal sentido, debido a la problemática antes mencionada que tienen las microempresas proveedoras de Sider Perú, se plantea la siguiente interrogante: ¿Cumplen las microempresas con los criterios de calificación en los Niveles de Homologación para ser consideradas empresas proveedoras de Sider PerúGerdau? Chimbote, Perú 2016?

Para dar respuesta a la pregunta planteada, se plantean los siguientes objetivos: Determinar el cumplimiento de los criterios de calificación en los Niveles de Homologación para ser consideradas empresas proveedoras de Sider PerúGerdau. Chimbote, Perú 2016?

Para alcanzar el objetivo general se planteó los siguientes objetivos específicos:

Determinar el cumplimiento de los criterios calificación del Primer Nivel de Homologación que deben tener las micro y pequeñas empresas para ser consideradas empresas proveedoras de Sider Perú- Gerdau, Chimbote, Perú, 2016

Determinar el cumplimiento de los criterios de calificación del Segundo Nivel de Homologación que deben tener las micro y pequeñas empresas para ser consideradas empresas proveedoras de Sider Perú-Gerdau, Chimbote, Perú, 2016.

Determinar el cumplimiento de los criterios de calificación del Tercer Nivel de Homologación que deben tener las micro y pequeñas empresas para ser consideradas empresas proveedoras de Sider Perú-Gerdau, Chimbote, Perú, 2016.

La investigación se justifica dado que permite conocer cuáles son los estándares de certificación de calidad deben tener las micro y pequeñas empresas para poder ser proveedor de la empresa siderúrgica Sider Perú-Gerdau.

Así también se indica que el siguiente artículo contiene: el contexto teórico, materiales y métodos empleados, los resultados y discusión de estos, las conclusiones y finalmente la bibliografía empleada.

\section{Contexto Teórico}

Las pequeñas y medianas empresas (pymes), tienen una gran importancia en la economía y particularmente en el empleo a nivel nacional y regional, tanto en los países industrializados como en los de menor grado de desarrollo. Las PYMES representan a nivel mundial el segmento de la economía que aporta el mayor número de unidades económicas $\mathrm{y}$ personal ocupado; de ahí la relevancia de este tipo de empresas y la necesidad de fortalecer su desempeño, al incidir éstas de manera fundamental en el comportamiento global de la economía nacional. Son muchas las características comunes que tienen las pequeñas $y$ microempresas a nivel mundial, entre ellas 
tenemos: utilizan más maquinaria y equipo, aunque se sigan basando más en el trabajo que en el capital; dominan y abastecen un mercado más amplio, aunque no necesariamente tiene que ser local o regional, ya que muchas veces llegan a producir para el mercado nacional e incluso para el mercado internacional; están en proceso de crecimiento, la pequeña tiende a ser mediana y está aspira a ser grande; obtienen algunas ventajas fiscales por parte del Estado que algunas veces las considera causantes menores dependiendo de sus ventas y utilidades; su tamaño es pequeño o mediano en relación con las otras empresas que operan en el ramo. Sin embargo, a pesar de la gran importancia que tienen estas unidades de negocios en la economía de un país, en su gran mayoría, tienen aún muchas dificultades para ingresar a competir en mercados internacionales, fundamentalmente, por su falta de competitividad.

Es así como, en un mundo cada vez más globalizado, donde hay necesidad de contar con organizaciones empresariales competitivas, un enfoque de un sistema de gestión de calidad total se convierte en una filosofía de la alta dirección que le va a permitir a la organización una permanencia en el mercado enfocada en el largo plazo. Como lo señala James (2000), un sistema de gestión de calidad total es una filosofía de dirección generada por una orientación práctica, que concibe un proceso que visiblemente ilustra un compromiso de crecimiento y supervivencia organizativa. Significa acción enfocada hacia la mejora de la calidad en el trabajo y a la organización como un todo. Permite a una organización, por medio de una estrategia coordinada de trabajo en equipo y de innovaciones, satisfacer las expectativas y necesidades del cliente.

Las organizaciones empresariales competitivas están basando el accionar de sus procesos, dese el inicio de la cadena de suministros, es decir, desde sus proveedores. Pinedo (2008), indica, Hace tiempo los japoneses se refieren a la importancia de la colaboración con los proveedores para el éxito de sus negocios. Más aún, en aquellas industrias como las confecciones, minería y otras; el poder de los proveedores ha llegado a cambiar la cadena de suministros.

En una organización empresarial se deben utilizar adecuadas estrategias para identificar a sus proveedores y luego mantener con ellos lazos de comunicación en el corto, mediano y largo plazo. El proveedor debe convertirse en un aliado estratégico que permita a la empresa su permanencia en el mercado. En tal sentido, hay necesidad de contar con una oportuna comunicación entre el proveedor y la empresa para que el cliente pueda obtener un producto o servicio en las mejores condiciones posibles. La oportuna comunicación que exista entre un proveedor y la empresa generarán relaciones mutuamente beneficiosas que permitirán aumentar la capacidad de ambos para crear valor. Todo lo contrario, sucederá si no existe una comunicación fluida y oportuna que finalmente el cliente externo lo percibirá como una mala atención. Es por ello, la gran importancia de identificar bien al proveedor para conocer si tiene los insumos o materiales 
necesarios para poder producir, fabricar o elaborar a tiempo los productos/ servicios que necesita el mercado. Así mismo, hay una gran necesidad de contar con la información necesaria respecto a los costos de los materiales o insumos que provean. Así también, se necesita saber, de parte de los proveedores, la posibilidad de brindarle al cliente valores agregados relacionados con la promoción del producto o servicio. En suma, es importante que se mantenga con el proveedor, una constante comunicación para hacer más accesible la cadena de suministros. Por ello, la empresa debe realizar una buena gestión con sus proveedores a través de una adecuada selección, evaluación y seguimiento para que los resultados de sus procesos se realicen de la mejor manera posible.

$\begin{array}{ccc}\text { En el contexto económico } & \text { y } \\ \text { empresarial actual, donde } & \text { la }\end{array}$
competitividad se da en todo tipo de organización empresarial, hay necesidad de contar con un valor agregado que permita una diferenciación la misma que se obtienen a través de la eficiencia y eficacia empresarial. Muchas empresas han optado por externalizar o subcontratar sus actividades concentrándose sólo en aquellas que son propias del "core" del negocio que le permitan generar un valor agregado a sus clientes. La estrategia de externalización o subcontratación permite que se obtengan mejores costos, pero, además, permite detectar oportunidades de negocios en beneficios de las micro y pequeñas empresas de un determinado territorio. Sin embargo, no solo basta con tener los costos más bajos sino también se deben tener en cuenta la calidad, precio, entrega, experiencia, crédito, entre otros aspectos por lo que hay una gran necesidad de tener una relación más estrecha con los proveedores. Pinedo (2008) señala que de la necesidad de establecer relaciones más estrechas con los proveedores hace necesario llegar a un conocimiento sobre el mismo.

A lo largo del proceso de homologación de un proveedor se consigue una visión real del grado de adecuación del proveedor a las necesidades del cliente. Así también, Díaz y Jungbluth (1998), indican que, de acuerdo al tipo de empresa, sus necesidades y sus relaciones, tanto con los clientes con proveedores, se crea un sistema de calidad, un método de trabajo, una estructura de la organización, que aseguran el cumplimiento de los requisitos especificados. Las normas ISO de la serie 9000 y las equivalentes europeas detallan los elementos a tener en cuenta para implantar un sistema de calidad.

En el Perú, existen empresas que han venido homologando a sus proveedores a través de entidades certificadoras, basados en la norma ISO 9001: 2008. Una de ellas es Certificaciones del Perú S.A., CERPER, empresa privada con una trayectoria de cuatro décadas, dedicada a ofrecer servicios de inspección, muestreo, ensayos, certificación de productos y de sistemas de gestión, quien a través de su programa CERTIPYME, programa de enfoque empresarial, que reconoce progresivamente el nivel de competitividad de una Micro y Pequeña Empresa, mediante una certificación progresiva emitida. Dichos niveles son las siguientes: 
Nivel 1: Estructura Organizacional: Certificación de organización.

Nivel 2: Proceso y Producto principal: Certificación de proceso.

Nivel 3: Planeamiento Integral: Certificación de gestión.

Alcanzado el Tercer Nivel, la micro o pequeña empresa está en la capacidad de poder acceder a una certificación ISO.

\section{Materiales y métodos}

Diseño del estudio. El diseño del estudio fue: No experimental- transversal.

Fue no experimental, porque se realizó sin manipular deliberadamente la información que proporcionaron las empresas proveedoras de la Empresa Siderúrgica Sider Perú en estudio, sin sufrir ningún tipo de modificaciones.

Fue transversal, porque el estudio se realizó en un espacio de tiempo determinado porque tuvo un inicio y un fin es decir en el año 2016

Población y muestra. Se utilizó una población de 45 empresas proveedoras de la Empresa Siderúrgica Sider Perú Gerdau.

Se utilizó como muestra las 45 empresas proveedoras que decidieron mejorar sus niveles empresariales contribuyendo a su propio crecimiento y desarrollo.

Técnicas e instrumentos. La técnica que se utilizó fue la encuesta, porque es una técnica que se utilizó para recoger información de las empresas en estudio. El instrumento que se utilizó para registrar la información fue el cuestionario el cual estuvo estructurado con interrogantes según la norma ISO 9001:2008 sobre gestión de calidad, misión, visión, políticas, procesos, productos, clientes y proveedores para homologar a las empresas en los diferentes niveles empresariales.

Plan de Análisis. La metodología desarrollada para aplicar la homologación de proveedores, para el caso de las microempresas ubicadas en la ciudad de Chimbote está supeditada a lo señalado por el programa CERTIPYME en la que se contempla un conjunto de actividades que contribuyen a certificar empresas y homologarlas de acuerdo a las normas establecidas en la Norma ISO 9001:2008 Sistemas de Gestión de Calidad. Las fases del proceso de homologación contemplan las siguientes actividades: Diagnóstico, Capacitación, Asistencia técnica y/o Asesoría para la implementación de planes de mejora establecidos en el diagnóstico, Auditoría calificada del nivel alcanzado, y finalmente, Certificación emitida por empresa certificadora Certificaciones del Perú- CERPER.

a) Fase de Autodiagnóstico: En esta fase el empresario evalúa la situación de su empresa respondiendo los Criterios de Calificación de Certificación de Pymes del Nivel correspondiente.

b) Fase de Capacitación: Participan el Facilitador y el empresario. En esta fase, se realizan una serie de eventos académicos que permiten que el empresario pueda identificar y luego aplicar, en su empresa, los criterios de 
calificación llevados a cabo en el autodiagnóstico.

c) Fase de Consultoría: Aquí participan el consultor-asesor y el empresario para luego, a través de un proceso de acompañamiento, se pueda poner en práctica todos los criterios de calificación del Nivel de Certificación correspondiente.

d) Fase de Auditoría: Participa el Auditor de la empresa certificadora para dar fe de que los criterios de calificación del nivel correspondiente han sido aplicados en la empresa.

e) Fase de Certificación: Es la fase en la que se certifica a la empresa otorgándole a la empresa un certificado y premio según el puntaje obtenido como producto de haber cumplido con los criterios de calificación de certificación.

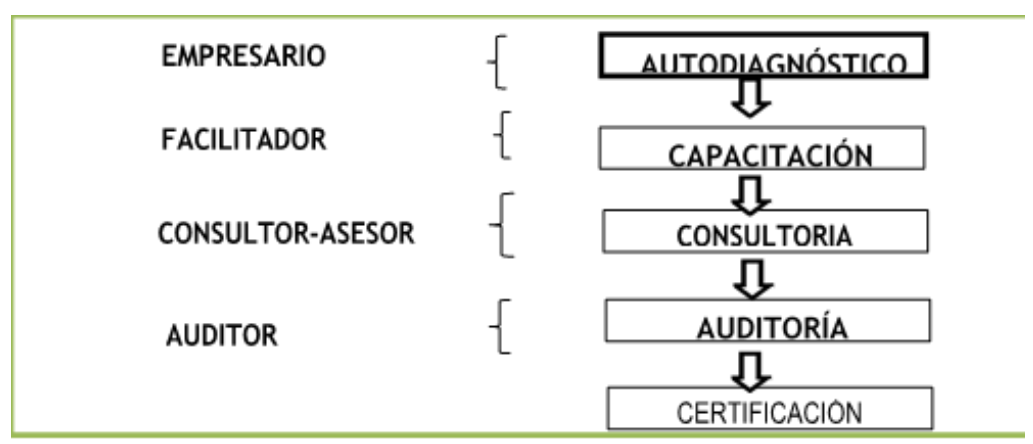

Figura 1: Fases del proceso de Homologación. Elaboración propia.

Niveles de Homologación

El Programa CERTIPYME tiene un esquema de certificación para desarrollar empresas confiables y competitivas. Certipyme es un sistema de evaluación y certificación, el cual permite a las empresas demostrar su capacidad en el nivel alcanzado, desarrollado en base a los modelos del sistema ISO, el cual busca elevar la competitividad de la micro, pequeña y mediana empresa a través de un proceso progresivo. El certipyme fue desarrollado por el Consorcio COPEME y Quality Consulting participando como empresa certificadora CERPER SA: El proceso se desarrolla en tres niveles:

Nivel I: Organización. En este nivel se consideran los siguientes criterios:

1. Sistema de gestión de calidad, 2.Experiencia,

3.Finanzas, 4.Organización y personal de la empresa, 5.Infraestructura de la empresa, 6.Logística de entrada, 7.Proceso logístico complementario, 8.Proceso principal del negocio.

Nivel II: Proceso. En este nivel se consideran los siguientes criterios:

a. Logística de entrada. a.1 gestión de proveedores, a. 2 compras.

b. Proceso logístico complementario. b.1 gestión de almacenes, b.2 despacho de productos.

c. Proceso principal del negocio. c.1. operación, c.2. mantenimiento $\mathrm{y}$ calibración, c.3. seguridad,

d. Marketing y ventas, d.1. comercialización, d.2. relación con el cliente.

Nivel III: Gestión. En este nivel se consideran los siguientes criterios:

1. Producción y prestación del servicio, 2. Identificación y trazabilidad, 3. Propiedad el cliente, 4. Preservación del producto, 5. Control de los equipos de seguimiento y medición, 6 . Seguimiento y medición. 


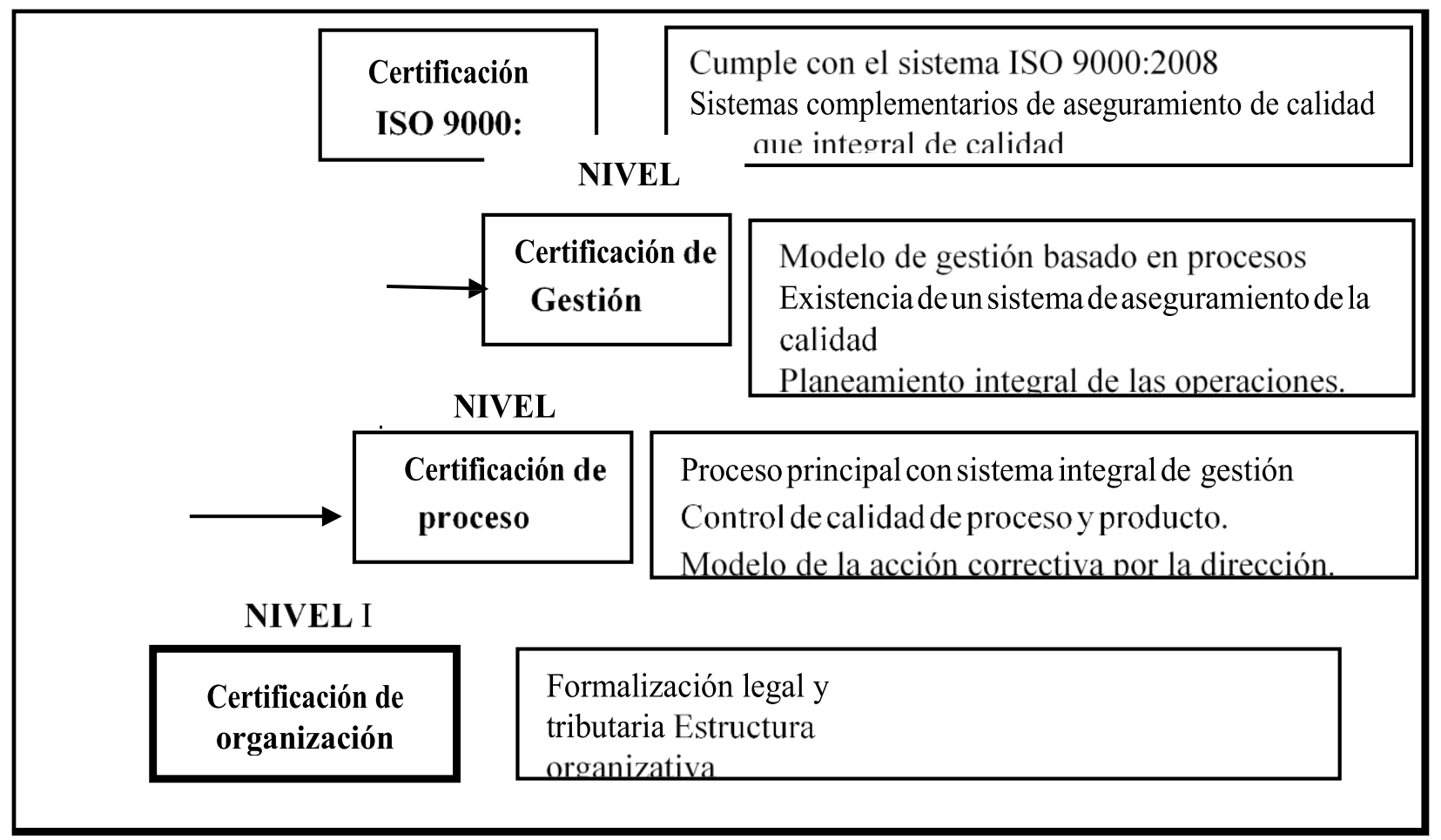

Figura 2: Niveles de certificación. Elaboración propia.

\section{Resultados}

Al momento de realizar el registros de personal. El $100 \%$ de las Autodiagnóstico, El 100\% de las empresas no identifican su filosofía organizacional la cual se expresa en la visión, misión, políticas y objetivos de calidad. Así también, el $95.56 \%$ no tiene elaborado su mapa de procesos. Sólo el $17.78 \%$ tiene empresas, no tiene un Plan de producción, tampoco elaboran instrucciones de trabajo, no tienen definidas sus responsabilidades de su proceso principal. Sólo el $17.78 \%$ lleva a cabo el control del producto terminado.

\section{Criterios de Calificación de Certificación de pymes al Primer Nivel}

\begin{tabular}{llllll}
\hline Criterios & \multicolumn{2}{l}{ Conforme } & \multicolumn{2}{c}{ No cumple } & Total \\
& $\mathrm{N}^{\circ}$ & $\%$ & $\mathrm{~N}^{\circ}$ & $\%$ & \\
\hline Visión & 0 & 0 & 45 & 100.00 & 45 \\
Misión & 0 & 0 & 45 & 100.00 & 45 \\
Comunicación de la visión y misión & 0 & 0 & 45 & 100.00 & 45 \\
Políticas de Calidad & 0 & 0 & 45 & 100.00 & 45 \\
Objetivos de calidad son medibles y & 0 & 0 & 45 & 100.00 & 45 \\
coherentes & & & & &
\end{tabular}




\begin{tabular}{llllll}
\hline Criterios & \multicolumn{2}{l}{ Conforme } & \multicolumn{2}{l}{ No cumple } & Total \\
& $\mathrm{N}^{\circ}$ & $\%$ & $\mathrm{~N}^{\circ}$ & $\%$ & \\
\hline Comunicación y entendimiento: Política & & & & & \\
y Objetivos de Calidad & 0 & 0 & 45 & 100.00 & 45 \\
Mapa de Procesos & 2 & 4.44 & 43 & 95.56 & 45 \\
Documentado que define controles & 2 & 4.44 & 43 & 95.56 & 45 \\
Manejo de registros adecuado & 0 & 0.00 & 45 & 100.00 & 45 \\
Procesos de comunicación apropiados & 0 & 0.00 & 45 & 100.00 & 45 \\
Crédito de la empresa & 45 & 100.00 & 0 & 0.00 & 45 \\
Registros de personal & 8 & 17.78 & 37 & 82.22 & 45 \\
Plan de Producción & 0 & 0.00 & 45 & 100.00 & 45 \\
$\quad$ Instrucciones de Trabajo & 0 & 0.00 & 45 & 100.00 & 45 \\
Responsabilidades definidas de su & & & & & \\
proceso principal & 0 & 0.00 & 45 & 100.00 & 45 \\
Control de Producto Terminado & 8 & 17.78 & 37 & 82.22 & 45 \\
\hline
\end{tabular}

Fuente: El autor

\section{Criterios de Calificación de Certificación de pymes al Segundo Nivel}

Sólo el $19.23 \%$ de las empresas cumple parcialmente con seleccionar a sus proveedores. El 53\% cumple parcialmente con tener un Plan de compras. El 69\% no cumple con definir sus especificaciones de materia prima e insumos críticos. Sólo el 11\% cumple parcialmente con tener procedimientos escritos e implementados. Así también, el $46 \%$ de las empresas no cumple con tener procedimientos de seguridad escritos e implementados.

\begin{tabular}{|c|c|c|c|c|c|c|c|c|c|}
\hline \multirow[t]{2}{*}{ Criterios } & \multicolumn{2}{|c|}{ Conforme } & \multicolumn{2}{|c|}{ Parcial 2} & \multicolumn{2}{|c|}{ Parcial 1} & \multicolumn{2}{|c|}{ No Cumple } & \multirow[t]{2}{*}{ Total } \\
\hline & $\mathrm{N}^{\circ}$ & $\%$ & $\mathrm{~N}^{\circ}$ & $\%$ & $\mathrm{~N}^{\circ}$ & $\%$ & $\mathrm{~N}^{\circ}$ & $\%$ & \\
\hline $\begin{array}{l}\text { La empresa selecciona a sus } \\
\text { proveedores }\end{array}$ & 0 & 0.00 & 2 & 7.69 & 5 & 19.23 & 19 & 73.08 & 26 \\
\hline Plan de compras & 0 & 0.00 & 8 & 30.77 & 6 & 23.08 & 12 & 46.15 & 26 \\
\hline Ha definido especificaciones de Materia & & & & & & & & & \\
\hline Prima e insumos críticos & 5 & 19.23 & 1 & 3.85 & 2 & 7.69 & 18 & 69.23 & 26 \\
\hline Sistema de transporte adecuado & 2 & 7.69 & 1 & 3.85 & 2 & 7.69 & 21 & 80.77 & 26 \\
\hline $\begin{array}{l}\text { Proceso principal en un manual de } \\
\text { procesos }\end{array}$ & 0 & 0.00 & 0 & 0.00 & 2 & 7.69 & 24 & 92.31 & 26 \\
\hline $\begin{array}{l}\text { Capacitación para más del } 85 \% \text { de los } \\
\text { puestos existentes }\end{array}$ & 0 & 0.00 & 2 & 7.69 & 3 & 11.54 & 21 & 80.77 & 26 \\
\hline Especificaciones para más del $85 \%$ de & & & & & & & & & \\
\hline los productos terminados & 0 & 0.00 & 3 & 11.54 & 5 & 19.23 & 18 & 69.23 & 26 \\
\hline $\begin{array}{l}\text { Procedimientos escritos e } \\
\text { implementados }\end{array}$ & 0 & 0.00 & 0 & 0.00 & 3 & 11.54 & 23 & 88.46 & 26 \\
\hline $\begin{array}{l}\text { Procedimientos de seguridad escritos e } \\
\text { implementados }\end{array}$ & 0 & 0.00 & 5 & 19.23 & 9 & 34.62 & 12 & 46.15 & 26 \\
\hline Procedimientos de toma de pedidos & 0 & 0.00 & 2 & 7.69 & 3 & 11.54 & 21 & 80.77 & 26 \\
\hline
\end{tabular}

Fuente. El autor 
Criterios de Calificación de Certificación de pymes al Tercer Nivel

\begin{tabular}{|c|c|c|c|c|c|c|c|c|c|}
\hline \multirow[t]{2}{*}{ Criterios } & \multicolumn{2}{|c|}{ Conforme } & \multicolumn{2}{|c|}{ Parcial 2} & \multicolumn{2}{|c|}{ Parcial 1} & \multicolumn{2}{|c|}{ No Cumple } & \multirow[t]{2}{*}{ Total } \\
\hline & $\mathrm{N}^{\circ}$ & $\%$ & $\mathrm{~N}^{\circ}$ & $\%$ & $\mathrm{~N}^{\circ}$ & $\%$ & $\mathrm{~N}^{\circ}$ & $\%$ & \\
\hline $\begin{array}{l}\text { Especificaciones técnicas del producto } \\
\text { y/o servicio }\end{array}$ & 3 & 12.50 & 12 & 50.00 & 9 & 37.50 & 0 & 0.00 & 24 \\
\hline Instrucciones de trabajo del personal & 2 & 8.33 & 6 & 25.00 & 5 & 20.83 & 11 & 45.83 & 24 \\
\hline $85 \%$ de equipos de uso son adecuado & 2 & 8.33 & 6 & 25.00 & 12 & 50.00 & 4 & 16.67 & 24 \\
\hline $\begin{array}{l}\text { Uso de equipos de seguimiento y } \\
\text { medición }\end{array}$ & 2 & 8.33 & 8 & 33.33 & 9 & 37.50 & 5 & 20.83 & 24 \\
\hline $\begin{array}{l}\text { Plan de producción y hace una medición } \\
\text { del seguimiento }\end{array}$ & 0 & 0.00 & 1 & 4.17 & 3 & 12.50 & 20 & 83.33 & 24 \\
\hline $\begin{array}{l}\text { Actividades de liberación, entrega y } \\
\text { posteriores a la entrega }\end{array}$ & 0 & 0.00 & 5 & 20.83 & 9 & 37.50 & 10 & 41.67 & 24 \\
\hline $\begin{array}{l}\text { Procedimientos escritos para } \\
\text { identificación de recursos }\end{array}$ & 0 & 0.00 & 8 & 33.33 & 11 & 45.83 & 5 & 20.83 & 24 \\
\hline Requisitos de seguimiento y medición & 0 & 0.00 & 8 & 33.33 & 11 & 45.83 & 5 & 20.83 & 24 \\
\hline $\begin{array}{l}\text { Control y seguimiento en las etapas del } \\
\text { proceso de producción }\end{array}$ & 0 & 0.00 & 8 & 33.33 & 11 & 45.83 & 5 & 20.83 & 24 \\
\hline $\begin{array}{l}\text { Evidencias de diseño, y registros de } \\
\text { trazabilidad }\end{array}$ & 0 & 0.00 & 8 & 33.33 & 9 & 37.50 & 7 & 29.17 & 24 \\
\hline $\begin{array}{l}\text { Cuidado de los bienes de clientes en } \\
\text { manual de procesos }\end{array}$ & 0 & 0.00 & 0 & 0.00 & 0 & 0.00 & 24 & 100.00 & 24 \\
\hline $\begin{array}{l}\text { Procedimientos para identificación y } \\
\text { manipulación de productos }\end{array}$ & 8 & 33.33 & 6 & 25.00 & 5 & 20.83 & 5 & 20.83 & 24 \\
\hline
\end{tabular}

Fuente. El autor

El $87.50 \%$ de las empresas cuenta con las Especificaciones técnicas del producto y/o servicio. Sólo el $45.83 \%$ de las empresas cuentan con Instrucciones de trabajo del personal. Sólo el 8.33 hace uso de equipos de seguimiento y medición. El 78.33 utiliza procedimientos escritos para identificación de sus recursos. El 100\% de las empresas no cumple con el cuidado de los bienes de clientes que se encuentren especificados en un Manual de Procesos.

Nivel de cumplimiento de los criterios de calificación obtenidos luego de la Fase de Consultoría del Proceso de Homologación fueron las siguientes:

\section{Primer nivel}

\begin{tabular}{|l|l|l|}
\hline Cumplimiento & $\mathrm{N}^{\circ}$ De Empresas & $\%$ \\
\hline $\mathrm{Si}$ & 45 & 100 \\
\hline No & 0 & \\
\hline TOTAL & 45 & 100 \\
\hline
\end{tabular}

El $100 \%$ de las empresas que participaron en el Primer Nivel cumplieron con los criterios de calificación del proceso de homologación

\section{Segundo nivel}

\begin{tabular}{|l|l|l|}
\hline Cumplimiento & $\mathrm{N}^{\circ}$ De Empresas & $\%$ \\
\hline $\mathrm{Si}$ & 26 & 58 \\
\hline No & 19 & 42 \\
\hline TOTAL & 45 & 100 \\
\hline
\end{tabular}


Del $100 \%$ de empresas que participaron en el Segundo Nivel, el 58\% cumplieron con los criterios de calificación del proceso de homologación.

\section{Tercer nivel}

\begin{tabular}{|l|l|l|}
\hline Cumplimiento & $\mathrm{N}^{\circ}$ De Empresas & $\%$ \\
\hline $\mathrm{Si}$ & 24 & 92 \\
\hline No & 2 & 8 \\
\hline TOTAL & 26 & 100 \\
\hline
\end{tabular}

Del $100 \%$ de empresas que pasaron del Segundo al Tercer Nivel, el 92\% cumplieron con los criterios de calificación del proceso de homologación.

\section{Discusión}

La discusión se enfoca a los resultados obtenidos después de la fase de Autodiagnóstico del Proceso de Homologación.

Relacionado con los criterios de calificación de certificación de pymes al Primer Nivel La empresa tiene definida su visión: El 100\% de las empresas no tiene definida su visión porque no conocen la filosofía organizacional en la cual está inmersa la visión, misión.

La empresa tiene definida su misión: El $100 \%$ de las empresas no tienen definida su misión. Dado que las empresas en estudio están administradas por sus propios dueños y la mayoría de ellos no tienen conocimientos empresariales por lo cual no realizan una planificación estratégica de sus empresas dentro de las cuales se encuentra la misión que es el propósito y lo que quiere llegar a ser la empresa eso se evidencia en que trabajan por simple rutina sin tener una meta que deseen lograr.

La empresa elabora su Mapa de Procesos: Estratégicos, Clave, Soporte, Medición y Mejora: El $95.56 \%$ de las empresas no cumple con elaborar su Mapa de Procesos: Estratégicos, Clave, Soporte, Medición y Mejora. Estas empresas no elaboran un mapa de procesos porque ellos no conocen la estructura de los mapas de procesos pero mencionan que saben los procedimientos que deben realizar para poder adquirir materia prima, insumos, procesos de elaboración de productos y procesos de ventas y solo dos empresas si las realizan porque esto les permite reducir algunos cuellos de botella, porque incrementa la participación y compromiso de los empleados dado que cada quien conoce cuáles son sus funciones $\mathrm{y}$ responsabilidades dentro de los procesos de adquisición de materia prima, elaboración, ventas y traslado de los productos hacia los clientes finales además esto les permite a las empresas evaluar la eficiencia, eficacia y productividad de las empresas.

La empresa en que régimen tributario se encuentra: El 100\% de las empresas encuestadas se encuentran en el régimen tributario general, por lo tanto se encuentran dónde están todas aquellas personas naturales, personas jurídicas, sucesiones indivisas, sociedades conyugales que opten tributar como tal y las asociaciones de hecho de profesionales y similares, además estas empresas llevan libros contables ( $\mathrm{Si}$ los ingresos son menores a 100 UIT, deberá llevar Registro de Ventas e Ingresos, Registro de Compras, Libro de Inventario y Balance, 
Libro de Caja y Bancos y Regiros de Activos Fijos. De ser mayores a 100 UIT, deberá llevar contabilidad completa. A su vez emiten comprobantes de pago en las ventas o servicios y solicitarlos en las compras que realice. Puede emitir los siguientes comprobantes de pago: Facturas, boleta de ventas, tickets, liquidación de compras, nota de crédito, nota de débito, guías de remisión, entre otros. Presentan la declaración de pagos mensuales y la declaración jurada anual del Impuesto a la Renta en la forma, plazo y lugar que la SUNAT establezca y efectúan retenciones a sus trabajadores (dependientes e independientes) y otras acciones que señala la ley.

La empresa mantiene algún tipo de registro de su personal: El $82.22 \%$ de las empresas no mantiene algún tipo de registro de su personal porque no tienen el área denominada escalafón que se encarga de mantener una ficha del personal con la finalidad de servir como una fuente de información necesaria para determinar la situación actual del colaborador, y para la toma de decisiones en cuanto a: Promoción o ascenso otorgar derechos conforme a ley, compensación por tiempo de servicio, movimiento de rotación o reubicación en las diferentes áreas de la empresa o conocer los datos personales de los trabajadores tales como: Dirección, números telefónicos, etc. Para poder ubicarlos en cualquier eventualidad $\mathrm{O}$ necesidad de su colaboración.

Relacionado con los criterios de calificación de certificación de pymes al segundo nivel, La empresa selecciona a sus proveedores en base a la calidad de los insumos: $\mathrm{El} 73.08 \%$ de las empresas no selecciona a sus proveedores en base a la calidad de los insumos, porque ellos trabajan en función a los proveedores porque los productos los obtienen a crédito y les pagan cuando ellos cobran. En síntesis, las empresas no cuentan con un capital importante que les permita seleccionar a sus proveedores porque si lo hacen los nuevos proveedores les van a exigir el pago al contado porque no los conocen y como no se cuenta con liquidez suficiente se tienen que conformar con seguir con sus antiguos proveedores.

La empresa dispone de un plan de compras generado con una frecuencia adecuada al giro y volumen de su negocio: El $46.15 \%$ de las empresas no dispone de un plan de compras generado con una frecuencia adecuada al giro y volumen de su negocio., el cual es controlado periódicamente por lo tanto eso hace que se compren insumos incompletos o que el volumen de compras sea mayor a lo que se necesita o viceversa generando pérdida de tiempo si se compra faltando y pérdida de dinero si se compra demasiado.

La empresa tiene un sistema de transporte adecuado para la entrega del producto: El $79.17 \%$ de las empresas no tienen un sistema de transporte adecuado para la entrega del producto porque no tienen idea de que esto es muy importante para la entrega de los productos dado que estos deben cumplir estándares de limpieza, seguridad, horas de conducción, control de la velocidad para no maltratar los productos frágiles garantizando que los productos se entreguen en buenas condiciones a los clientes. 
La empresa ha definido los requerimientos de capacitación para más del $85 \%$ de los puestos existentes: El $80.77 \%$ de las empresas no han definido los requerimientos de capacitación para más del $85 \%$ de los puestos existentes porque lo siguen considerando como un gasto porque la mayoría piensa que si capacita a los trabajadores estos más adelante se irán a trabajar a otras empresas y aportaran sus conocimientos en otras empresas y las que invirtieron en ellos seguirán careciendo de los aportes de conocimientos para su mejora continua.

La empresa posee procedimientos escritos e implementados: El $80.77 \%$ de las empresas no posee procedimientos escritos e implementados en cuanto a la toma de pedidos que realizan los clientes porque no utilizan tecnologías tales como: Software de ventas y pedidos, el cual es desarrollado para que los vendedores puedan tomar el pedido desde la web o cualquier dispositivo, además de llevar toda la información de los clientes de manera ordenada y encontrarla fácilmente minimizando los errores que se comenten al tomar los pedidos a mano en cuadernos.

Relacionado con los criterios de calificación de certificación de pymes al tercer nivel

La empresa cuenta con instrucciones de trabajo para más del $85 \%$ del personal que trabaja en las operaciones del producto/ servicios: El $45.83 \%$ de las empresas no cuenta con instrucciones de trabajo para más del $85 \%$ del personal que trabaja en las operaciones del producto/ servicios este porcentaje ha disminuido porque las empresas han entendido que es importante que sus trabajadores conozcan los procedimientos de trabajo en la realización de sus productos y/o servicios terminados para mejorar la satisfacción de sus clientes.

La empresa tiene definido su proceso de información, consultas y retroalimentación con el cliente: El $41.67 \%$ no tiene definido su proceso de información, consultas retroalimentación con el cliente. $\mathrm{La}$ mayoría de las empresas aun no tienen definido proceso de información, consultas y retroalimentación con los clientes eso dificulta la fidelización de los clientes porque no se tiene un sistema de atención para resolver dudas, consultas, etc. por falta de un diseño de procesos enfocados a atender las necesidades de los clientes.

Mantiene registros de lo informado al cliente sobre lo sucedido con los bienes de su propiedad: El $62.50 \%$ de las empresas no mantiene registros de lo informado al cliente sobre lo sucedido con los bienes de su propiedad porque no tienen un sistema de gestión enfocados en los clientes para asegurar sistemas de atención, cuidado de bienes y atención a sus consultas, sugerencias y reclamos.

La empresa tiene procedimientos escritos e implementados para la identificación y manipulación para preservar el producto durante el proceso interno de producción para mantener la conformidad con los requisitos: El 62.50\% de las empresas no tiene procedimientos escritos e implementados para la identificación y manipulación para preservar el producto durante el proceso 
interno de producción para mantener la conformidad con los requisitos dado que en el proceso de la elaboración del producto lo que a las empresas les importa es entregar los productos en el menor tiempo posible saltándose esos procesos para la conservación de los productos.

La empresa tiene procedimientos escritos e implementados de identificación y manipulación para preservar el producto durante la entrega al destino previsto para mantener la conformidad con los requisitos: El $33.33 \%$ de las empresas tiene procedimientos escritos e implementados de identificación $\mathrm{y}$ manipulación para preservar el producto durante la entrega al destino previsto para mantener la conformidad con los requisitos, porque de repente los productos que trasportan son delicados y si se manipula mal se deteriorará y los clientes no los aceptaran siendo una pérdida para las empresas dado que no solo gastarán en elaborarlos, sino que también pagan el transporte para el traslado y si son rechazados corren el riesgo de que los clientes en lo sucesivo ya no les compren, pero allí no queda todo porque con un cliente descontento no solamente significa perder a ese único cliente sino que también se perderá a los clientes potenciales porque el cliente descontento comunicará que ya no le compren a la empresa por problemas internos y porque los productos que ofrecen a la venta son entregados deteriorados.

b) La discusión se enfoca a los resultados obtenidos después de la fase de Consultoría del Proceso de Homologación. Del 100\% de empresas que participaron en el Primer Nivel, todas ellas cumplieron con los criterios de calificación y pasaron al Segundo Nivel. Del $100 \%$ de empresas que participaron en el Segundo Nivel, sólo el 58\% cumplieron con los criterios de calificación del proceso de homologación, significando que un $42 \%$ o se retiraron del proceso o no cumplieron con las exigencias solicitadas en este nivel. De las empresas que pasaron del segundo al tercer Nivel, el 92\% cumplieron con los criterios de calificación del proceso de homologación.

\section{Conclusiones}

Relacionado con el cumplimiento de los criterios de Criterios de calificación de certificación de mypes del Primer Nivel, Del $100 \%$ de empresas que participaron en el proceso de homologación, todas ellas cumplieron con los criterios de calificación y pasaron al segundo Nivel.

Relacionado con el cumplimiento de los criterios de Criterios de calificación de certificación de Mypes del Segundo Nivel

Del $100 \%$ de empresas que participaron en el proceso de homologación, sólo el 58\% de empresas cumplieron con los criterios de calificación y pasaron al Tercer Nivel.

Relacionado con el cumplimiento de los criterios de Criterios de calificación de certificación de Mypes del Tercer Nivel

Del $100 \%$ de empresas que
participaron en el proceso de
homologación en el Tercer Nivel, el $92 \%$
de empresas cumplieron con los criterios
de calificación del Proceso de
Homologación.




\section{Referencias}

Arenas, A. (2009). Sistema de gestión de la calidad según ISO-9000. Córdoba, AR: El Cid Editor | apuntes. Retrieved from http://www.ebrary.com

Cuatrecasas, A. L. (2000). Gestión de la calidad total. Sunny Isles Beach, US: Ediciones Díaz de Santos. Retrieved from http://www.ebrary.com

Díaz, B., \& Jungbluth, C. (1998). La Calidad Total en la empresa peruana. Proceso, resultados y perspectivas. Perú. Universidad de Lima.

Espinosa (2009). Calidad total. Córdoba, AR: El Cid Editor | apuntes. Retrieved from http://www.ebrary.com

James, P. (2000) Gestión de la Calidad Total. Un texto introductorio. Madrid: Prentice Hall Iberia.

Kannan, V. R., \& Tan, K. C. (2002). 'Supplier selection and assessment: Their impact on business performance'. Journal of Supply Chain Management, 38(3), 11-21.

Pinedo, N. (2008). Sistema de evaluación y Homologación de proveedores. Universidad Nacional Mayor de San Marcos. Recuperado el 18 de marzo del 2016 http://cybertesis.unmsm.edu.pe/bitstream/cyberte sis/1513/1/Pinedo_cn.pdf

Pinto, S. (2016). Los procesos de compra y la negociación con proveedores: una nueva forma de relación basada en la cooperación. Madrid, ESPAÑA: FC Editorial. Retrieved from http://www.ebrary.com

Ragatz, G. L., Handfield, R. B., \& Scannell, T. V. (1997). 'Success factors for integrating suppliers into new product development'. Journal of product innovation management, 14(3), 190202.

Stoner, J., Freeman, E. Gilbert, D. (2002). Administración. México. Prentice Hall Hispanoamericana, SA. 